\title{
p21-activated protein kinase 1 induces the invasion of gastric cancer cells through c-Jun NH2-terminal kinase-mediated activation of matrix metalloproteinase-2
}

\author{
LIANG-HUI LI ${ }^{1}$, GUO-YANG WU ${ }^{1}$, YI-ZHUO LU ${ }^{1}$, XUE-HUA CHEN ${ }^{2,3}$, BING-YA LIU ${ }^{2,3}$, \\ MIN-HUA ZHENG ${ }^{2-4}$ and JIAN-CHUN CAI ${ }^{1}$ \\ ${ }^{1}$ Department of General Surgery, Zhongshan Hospital, Xiamen University, Xiamen 361004; \\ ${ }^{2}$ Department of General Surgery, Ruijin Hospital, Shanghai Jiaotong University School of Medicine; \\ ${ }^{3}$ Shanghai Institute of Digestive Surgery; ${ }^{4}$ Department of Shanghai Minimally \\ Invasive Surgery, Ruijin Hospital, Shanghai 200025, P.R. China
}

Received October 13, 2016; Accepted December 13, 2016

DOI: $10.3892 /$ or. 2017.5643

\begin{abstract}
Gastric cancer (GC) is one of the most common malignancies worldwide. The prognosis of GC is poor, mostly due to widespread metastasis. p21-activated kinase 1 (Pak1), the best characterized member of an evolutionarily conserved family of serine/threonine kinases, plays an important role in the regulation of cell morphogenesis, motility, mitosis and angiogenesis. By qRT-PCR and Gelatin zymograph assay, we demonstrated in the present study that stable overexpression of Pak1 induced matrix metalloproteinase (MMP)-2 mRNA expression and activity in the human MKN45 GC cell line. Conversely, knockdown of endogenous Pak1 expression by small interfering RNA (siRNA) decreased MMP-2 mRNA expression and activity in the MKN45 GC cells. Activation of c-Jun N-terminal kinase (JNK) was required for Pak1induced upregulation of MMP-2 mRNA level and activity. Moreover, upregulation of MMP-2 by Pak1 via the JNK pathway notably promoted the invasion of MKN45 GC cells. Overexpression of MMP-2 mRNA was once again confirmed to be associated with GC metastasis. In conclusion, our results demonstrated for the first time that Pak1 stimulated MMP-2 mRNA expression and activity in MKN45 GC cells. The JNK signaling pathway was involved in Pak1 modulation of MMP-2, which was important for MKN45 GC cell invasiveness.
\end{abstract}

Correspondence to: Dr Jian-Chun Cai, Department of General Surgery, Zhongshan Hospital, Xiamen University, Xiamen 361004, P.R. China

E-mail:1lhxmzs@sina.com

Key words: gastric cancer metastasis, p21-activated protein kinase, c-Jun NH2-terminal kinase, matrix metalloproteinase-2

\section{Introduction}

Gastric cancer (GC) is one of the most common types of cancer worldwide, and is the second leading cause of cancer-related deaths (1). Despite unremitting efforts to improve a variety of diagnostic and therapeutic methods, tumor metastasis after surgery is still the greatest threat to patients. Thus, elucidation of the process and mechanism of GC metastases is required to improve the prognosis of GC patients.

p21-activated kinase 1 (Pak1) is the best characterized member of an evolutionarily conserved family of serine/threonine kinases (2), which plays a role in a variety of cellular functions such as cytoskeletal reorganization, cell motility, apoptosis and transformation (3-5). Pak1 has been identified as an effector molecule for the small GTPases Rho, Racl and Cdc42 (6). Amplification of Pak1 has been found in breast, renal, liver and colorectal cancer. Moreover, Pak1 has been reported to induce proliferation, motility and invasion of these cancer cells through its involvement in several cell signaling pathways, such as mitogen-activated protein kinases (MAPKs)

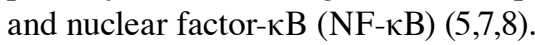

Matrix metalloproteinases (MMPs), a family of secreted or transmembrane enzymes, can collectively digest almost all extracellular matrix (ECM) and basement membrane components (9-11). Upregulation of MMPs has been reported to contribute to tumor cell invasion and metastasis, thus, leading to the development of malignant tumors (9-12). Both matrix metalloproteinase-2 and -9 (MMP-2 and MMP-9) which can degrade collagen IV, the major ECM component of basement membranes, have been found to be overexpressed in GC $(13,14)$. Studies have shown that high levels of MMP-2 and/or MMP-9 have a significant correlation with GC invasion $(14,15)$, thus, resulting in poor prognosis and shortening disease-free survival (15-17). MMP activity is closely controlled by physiological inhibitors, TIMPs including TIMP-1, $-2,-3$ and -4 (18).

Our previous study indicated that Pak1 was overexpressed in GC. Moreover, ectopic expression of Pak1 obviously increased the invasion of GC cells through phosphorylation of 
c-Jun N-terminal kinase (JNK) (19). In the present study, we sought to further examine the exact mechanism by which Pak1 stimulated the invasion of human GC cells. Our data revealed that Pak1 markedly enhanced MMP-2 mRNA expression and activity. Moreover, the JNK signaling pathway was involved in Pak1 upregulation of MMP-2 in MKN45 GC cells, which was important for the invasion of MKN45 GC cells. We reported for the first time that Pak1 induces the invasion of GC cells via the JNK-MMP-2 signaling pathway.

\section{Materials and methods}

Tissue samples and cell culture. Fifty-seven samples of primary GC tissues were obtained from the Shanghai Jiaotong University School of Medicine Ruijin Hospital. Fresh samples were harvested, and then, were immediately frozen in liquid nitrogen and kept at $-70^{\circ} \mathrm{C}$ until use. The study protocol was approved by the Medical Ethics and Human Clinical Trial Committee at the Shanghai Jiaotong University School of Medicine Ruijin Hospital. MKN45 human GC cells [American Type Culture Collection (ATCC) Manassas, VA, USA] were maintained in RPMI-1640 medium containing $10 \%$ fetal bovine serum (FBS).

Materials. Anti-Pak1 (N-20) (sc-882), anti-phospho-Pak1 (Thr423) (sc-12925), anti-DsRed (L-18) (sc-33353), anti-TIMP1 (sc-365905), anti-TIMP2 (sc-6835), anti-TIMP3 (sc-6836) and anti-TIMP4 (sc-9375) were obtained from Santa Cruz Biotechnology (Santa Cruz, CA, USA).

The anti-JNK and anti-phospho-JNK antibodies were purchased from Cell Signaling Technology Inc., (Beverly, MA, USA). The anti-glyceraldehyde-3-phosphate dehydrogenase (GAPDH) antibody was purchased from KangChen Bio-tech (Shanghai, China).

Constructs and production of a stable cell line. The Pak1 construct, pDs-Red2-Pak1, was a generous gift from Dr Jonathan Chernoff (Fox Chase Cancer Centre, Philadelphia, PA, USA). For constructing the stable transfectant, pDs-Red2 and pDs-Red2-Pak1 were separately transfected into MKN45 human GC cells using Lipofectamine 2000 (Invitrogen, Carlsbad, CA, USA) reagent according to the manufacturer's instructions. The transfected cells were selected in growth medium containing $1,000 \mu \mathrm{g} / \mathrm{ml}$ Geneticin (G418; Life Technologies, Grand Island, NY, USA). After 4-8 weeks, individual cell colonies were transferred for clone expansion and maintained in culture medium supplemented with $600 \mu \mathrm{g} / \mathrm{ml}$ G418.

Pak1 siRNA. For RNA interference (RNAi) of Pak1, Pak1 small interfering RNA and control siRNA were purchased from Santa Cruz Biotechnology. Each one (100 pmol in $2 \mathrm{ml}$ medium) was transfected into MKN45 human GC cells using Lipofectamine 2000.

Reverse transcription-PCR. Total RNA was extracted from samples using TRIzol reagent (Life Technologies), and $2 \mu \mathrm{g}$ of each RNA sample was used to prepare cDNA. The semi-quantitative PCR primer sequences for MMP-2 were: 5'-TAC AGG ATC ATT GGC TAC ACA CC (forward) and 5'-GGT CAC
ATC GCT CCA GAC T (reverse). The semi-quantitative PCR primer sequences for MMP-9 were: 5'-AGA CCT GGG CAG ATT CCA AAC (forward) and 5'-CGG CAA GTC TTC CGA GTA GT (reverse). Quantitative real-time PCR was carried out using the Applied Biosystems TaqMan system. Cellular 18S mRNA was used as an internal control.

Western blot analysis. Cells were harvested into RIPA lysis buffer (Pierce, Rockford, IL, USA) with a freshly added protease inhibitor cocktail and a phosphatase inhibitor cocktail (both from Roche Diagnostics, Mannheim, Germany). The cell lysate was cleared by centrifugation at $4^{\circ} \mathrm{C}$ and the supernatant was stored in small aliquots at $-80^{\circ} \mathrm{C}$. Normally, $20 \mu \mathrm{g}$ of sample was loaded into each lane, separated by SDS-polyacrylamide gel electrophoresis (SDS-PAGE), then transferred to a polyvinylidene difluoride membrane, and probed with respective antibodies. The density of blots was measured by PS software.

Gelatin zymography assay. For the zymography assay, cells $\left(2.5 \times 10^{5}\right)$ were seeded into 12 -well plates and incubated for $48 \mathrm{~h}$. Supernatants were collected and mixed with sample buffer followed by electrophoresis on a $10 \%$ SDS-PAGE containing $5 \mathrm{mg} / \mathrm{ml}$ of gelatin. The gel was washed with $2.5 \%$ Triton-X 100 solution for $2 \mathrm{~h}$ and further incubated in the reaction buffer $\left(50 \mathrm{mmol} / 1\right.$ Tris- $\mathrm{HCl}, 5 \mathrm{mmol} / 1 \mathrm{CaCl}_{2}, 1 \mu \mathrm{mol} / \mathrm{l}$ $\mathrm{ZnCl}_{2}$ and $1 \%$ Triton $\mathrm{X}-100$ ) for an additional $18 \mathrm{~h}$ at room temperature. The gel was then stained with $0.5 \%$ Coomassie blue for $9 \mathrm{~h}$, and subsequently immersed with destaining buffer (30\% methanol and $10 \%$ acetic acid) for $12 \mathrm{~h}$. Images were phtographed and the intensity of each band was digitally quantified.

Cell invasion assay. The polycarbonate membranes, $6.5-\mathrm{mm}$ in diameter with $8-\mu \mathrm{m}$ pores (Corning Costar, New York, NY, USA), coated with Matrigel (BD Biosciences, Bedford, MA, USA) were used for the invasion assay. Following the addition of medium containing $10 \%$ fetal calf serum (FCS) to the bottom chambers, single-cell suspensions in medium containing $0.1 \%$ BSA were seeded onto the filters $\left(1 \times 10^{5}\right.$ cells/ each well) and incubated for 24 or $48 \mathrm{~h}$ at $37^{\circ} \mathrm{C}$ in $5 \% \mathrm{CO}_{2}$. The filters were then washed and the cells on the upper surface were removed with cotton swabs. The cells that had invaded to the lower surface of the filter inserts were fixed with $5 \%$ paraformaldehyde for $15 \mathrm{~min}$ and stained with $0.1 \%(\mathrm{w} / \mathrm{v})$ crystal violet for $15 \mathrm{~min}$. The number of invaded cells was microscopically counted and 3 independent experiments were carried out to get an average cell number at a high magnification field.

Statistical analysis. Each experiment was duplicated at least 3 times. Results are presented as the mean \pm SE, and statistical comparisons were made using the Student's t-test, Fisher's exact or $\chi^{2}$ tests. Statistical SPSS version 15.0 was used to analyze data. Significance was defined at $\mathrm{P}<0.05$ levels.

\section{Results}

The effects of Pakl on the expression of MMP-2 and MMP-9 in human MKN45 GC cells. To elucidate the effects of Pak1 
A

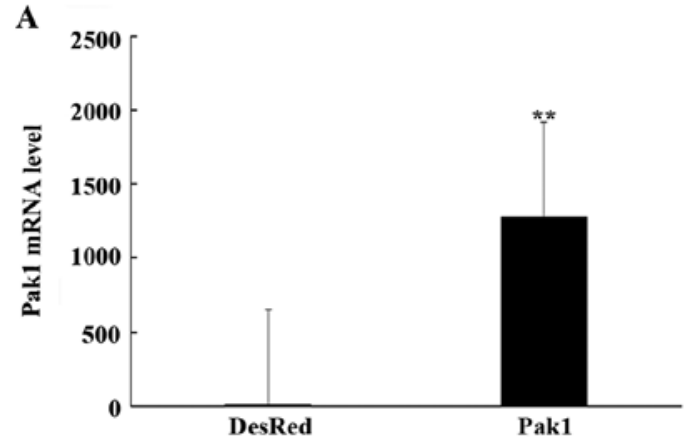

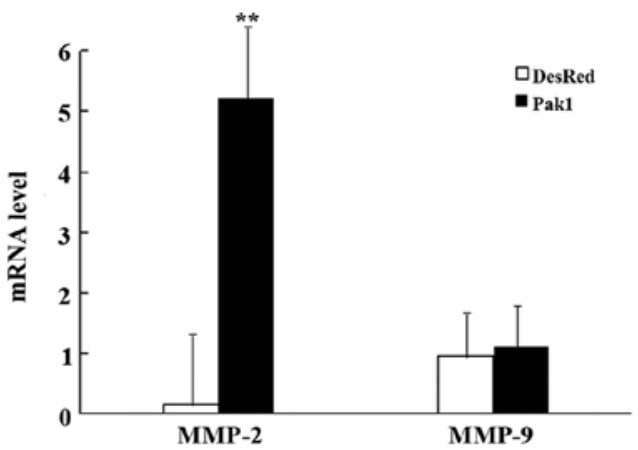

B
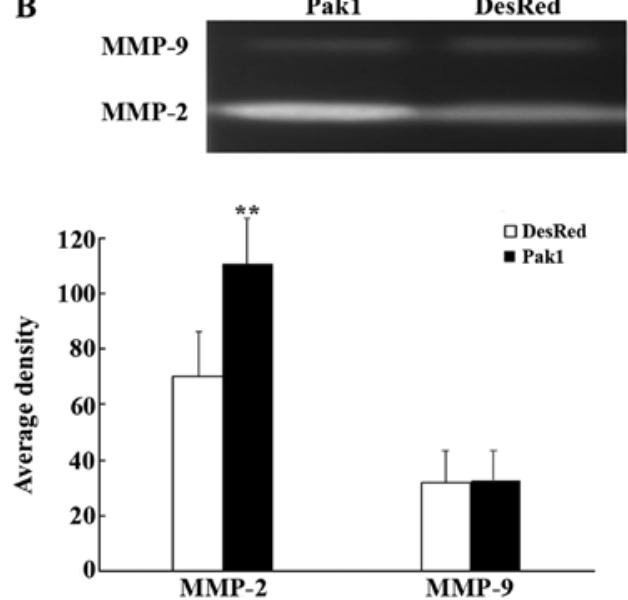

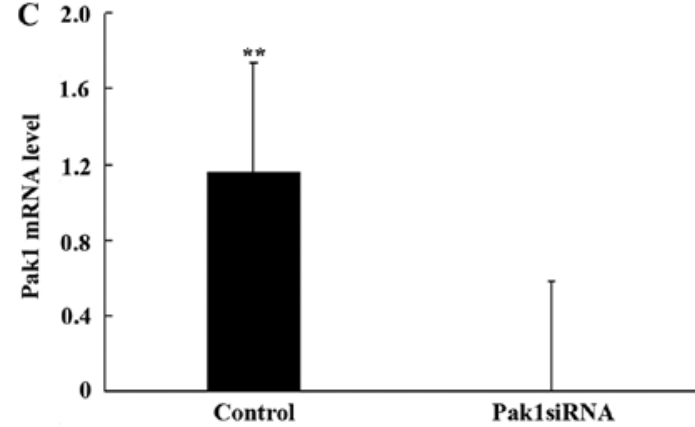

D
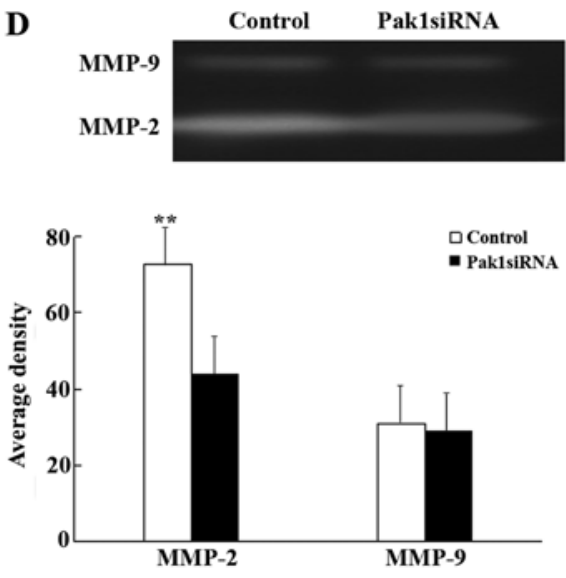

on the expression of MMP-2 and MMP-9 in GC, MKN45 GC cells were used to establish a stable cell line overexpressing the pDs-Red2 fusion form of Pak1. The stable transfectant cell line MKN45-Pak1 was established and confirmed with real-time

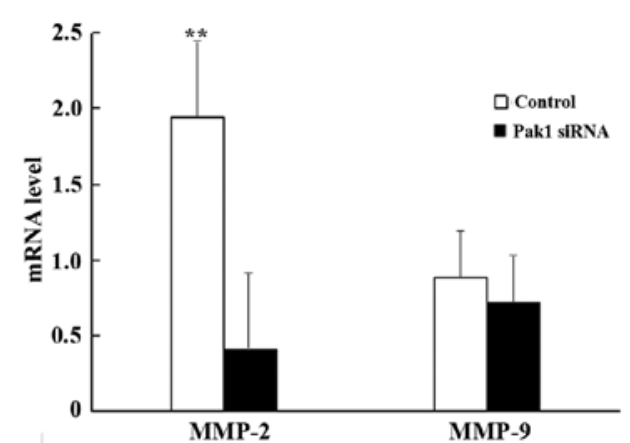

Figure 1. Effects of Pak1 on the expression of MMP-2 and MMP-9 in human MKN45 GC cells. (A) Quantitative real-time RT-PCR results of Pak1 mRNA in MKN45-DsRed2 and MKN45-Pak1 cells; ${ }^{* *} \mathrm{P}<0.01$ (left panel). Quantitative real-time RT-PCR results of MMP-2 and MMP-9 mRNA in MKN45-DsRed2 and MKN45-Pak1 cells; ${ }^{* *} \mathrm{P}<0.01$ (right panel). (B) Gelatin zymography assay results of MMP-2 and MMP-9 activities in MKN45-DsRed2 and MKN45-Pak1 cells (top panel). Quantification of MMP-2 and MMP-9 activities in MKN45-DsRed2 and MKN45-Pak1 cells; ${ }^{* *} \mathrm{P}<0.01$ (bottom panel). (C) Quantitative real-time RT-PCR results of Pak1 mRNA in MKN45 cells transfected with Pak1 siRNA and control cells; ${ }^{* *} \mathrm{P}<0.01$ (left panel). Quantitative real-time RT-PCR results of MMP-2 and MMP-9 mRNA in MKN45 cells transfected with Pak1 siRNA and control cells; ${ }^{* *} \mathrm{P}<0.01$ (right panel). (D) Gelatin zymography assay results of MMP-2 and MMP-9 activities in MKN45 cells transfected with Pak1 siRNA and control cells (top panel). Quantification of MMP-2 and MMP-9 activities in MKN45 cells transfected with Pak1 siRNA and control cells; ${ }^{* *} \mathrm{P}<0.01$ (bottom panel). Pak1, p21-activated kinase 1; MMP, matrix metalloproteinase; GC, gastric cancer.

quantitative reverse transcription PCR (qRT-PCR). The result revealed that Pak1 mRNA expression in the MKN45-Pak1 stable cell line was markedly higher than in the mock transfectant with pDsRed2 (named MKN45-DsRed2) $(\mathrm{P}<0.01$; 

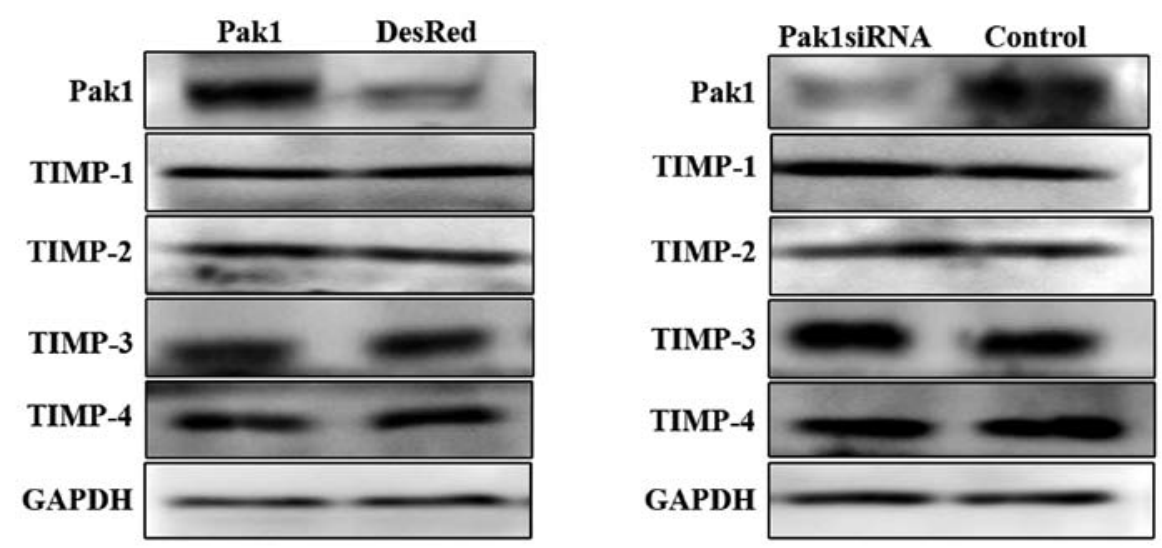

Figure 2. Effects of Pak1 on the expression of TIMPs in human MKN45 GC cells. Western blot analysis of TIMP-1, TIMP-2, TIMP-3 and TIMP-4 in MKN45DsRed2 and MKN45-Pak1 cells (left panel). Western blot analysis of TIMP-1, TIMP-2, TIMP-3 and TIMP-4 in MKN45 cells transfected with Pak1 siRNA and control cells (right panel). Pak1, p21-activated kinase 1; GC, gastric cancer.

Fig. 1A, left panel). We then determined the expression of matrix MMP-2 and -9 in the MKN45-DsRed2 and MKN45-Pak1 cells by qRT-PCR. Compared with the MKN45-DsRed2 cells, MMP-2 mRNA expression was significantly increased in the MKN45-Pak1 cells $(\mathrm{P}<0.01$; Fig. 1A). However, a significant difference in the mRNA level of MMP-9 between MKN45-Pak1 and the control cells was not found ( $\mathrm{P}=0.241$; Fig. 1A, right panel). We also performed gelatin zymography assay to assess the activities of MMP-2 and MMP-9 in the MKN45-DsRed2 and MKN45-Pak1 cells. Similarly, enhanced expression of Pak1 markedly increased MMP-2 activity ( $\mathrm{P}<0.01$; Fig. 1B), but had no effect on MMP-9 activity ( $\mathrm{P}=0.818$; Fig. 1B). To further confirm the effects of Pak1 on the expression of MMP-2 and MMP-9 in GC, we used RNAi to specifically knock down endogenous Pak1 in MKN45 cells. The specific knockdown of endogenous Pak1 in MKN45 cells was confirmed with qRT-PCR (Fig. 1C). We observed that MMP-2 mRNA expression and activity were sharply decreased in the MKN45 cells transfected with Pak1 siRNA ( $\mathrm{P}<0.01$, Fig. 1C; $\mathrm{P}<0.01$, Fig. 1D), but knockdown of endogenous Pak1 had no effects on MMP-9 mRNA expression and activity in the MKN45 cells ( $\mathrm{P}=0.063$, Fig. $1 \mathrm{C} ; \mathrm{P}=0.075$, Fig. 1D).

Effects of Pakl on the expression of TIMPs in human MKN45 $G C$ cells. Activation of MMPs is inhibited by TIMPs (18), therefore, we further examined whether the expression of TIMPs including TIMP-1, $-2,-3$ and -4 , was regulated by Pak1. Using western blot analysis, it was observed that overexpression of Pak1 or knockdown of endogenous Pak1 in MKN45 cells had no effects on the protein level of TIMPs (Fig. 2).

JNK mediates Pakl-induced upregulation of MMP-2 in human MKN45 GC cells. Our previous study revealed that activation of JNK was required for Pak1-mediated migration and invasion of GC cells (19). We therefore assessed whether JNK is involved in Pak1-upregulated MMP-2 in GC cells. Endogenous Pak1 was activated in MKN45-DsRed2 cells with epidermal growth factor (EGF) at $20 \mathrm{ng} / \mathrm{ml}$ at various times (0-40 $\mathrm{min})$, and then, lysates of these cells were subjected to western blotting with respective antibodies. We observed that JNK phosphorylation was increased in a time-dependent manner and a maximal increase was achieved at $30 \mathrm{~min}$, which was determined in our previous study (19) (Fig. 3A). To investigate whether MMP-2 expression was regulated by JNK phosphorylation, we evaluated the mRNA of MMP-2 and MMP-9 in MKN45-DsRed2 cells pretreated as in the aforementioned method by qRT-PCR. The present study revealed that MMP-2 mRNA expression was obviously increased in a time-dependent manner and a maximal increase was achieved at $30 \mathrm{~min}$, which was consistent with the increase of JNK phosphorylation (Fig. 3B). We also performed gelatin zymography assay to assess the activities of MMP-2 and MMP-9 in these cells. In accordance with changes in the mRNA expression, MMP-2 activity was observed to be enhanced in the same manner and a maximal increase was also achieved at $30 \mathrm{~min}$ (Fig. 3C). However, MMP-9 mRNA expression and activity were not found to be altered (Fig. 3B and C). In addition, we used JNK special inhibitor SP600125 in MKN45-Pak1 cells at various concentrations $(0,2.5,5,10$ and $20 \mu \mathrm{M}$ ) for $1 \mathrm{~h}$ to specifically inhibit the activity of JNK, and found that JNK phosphorylation was decreased in a concentration-dependent fashion and a maximal decrease was achieved at $10 \mu \mathrm{M}$ (Fig. 3D). The qRT-PCR result revealed that MMP-2 mRNA expression was sharply decreased in a concentration-dependent manner, and a maximal decrease was achieved at $10 \mu \mathrm{M}$, which was consistent with the downregulation of JNK phosphorylation (Fig. 3E). Gelatin zymography assay also revealed that MMP-2 activity was downregulated in the same way (Fig. 3F). MMP-9 mRNA expression and activity were not observed to be modified due to downregulation of JNK phosphorylation (Fig. 3E and F).

Upregulation of MMP-2 by Pakl via the JNK pathway is important for the invasion of human MKN45 GC cells. To further investigate the regulatory role of MMP-2 by the JNK pathway on GC cell invasion, an invasion assay was performed with MKN45-DesRed 2 cells incubated with EGF at $20 \mathrm{ng} / \mathrm{ml}$ for $30 \mathrm{~min}$, and the MKN45-DesRed2 cells were used as a control. Compared with the control, upregulation of MMP-2 expression by JNK enhanced cell invasion $(173.25 \pm 6.82$ vs. $69.75 \pm 7.78$ P<0.01; Fig. 4A). Activation of the JNK pathway and increase of MMP-2 activity were confirmed by western blotting and gelatin zymography assay, respectively (Fig. 4A). 
A
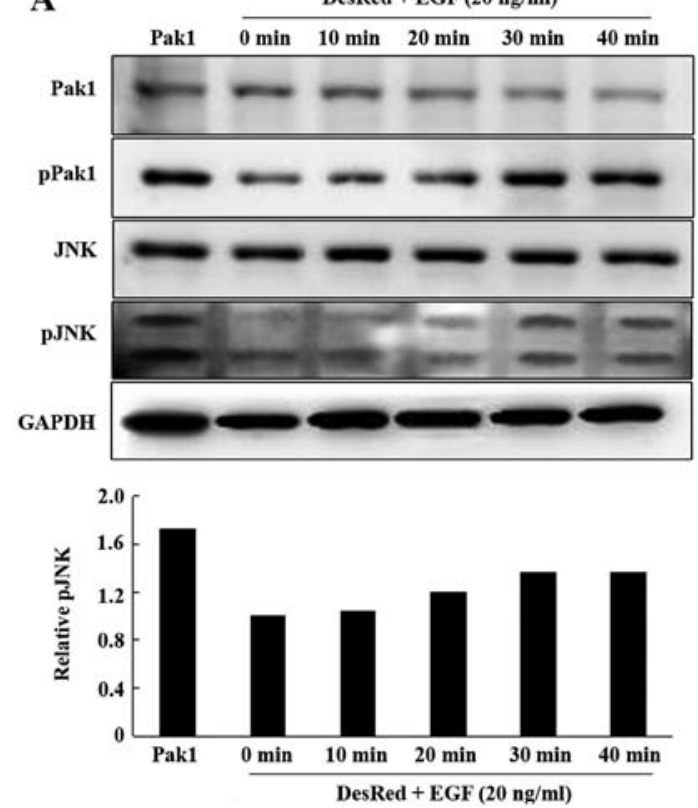

C
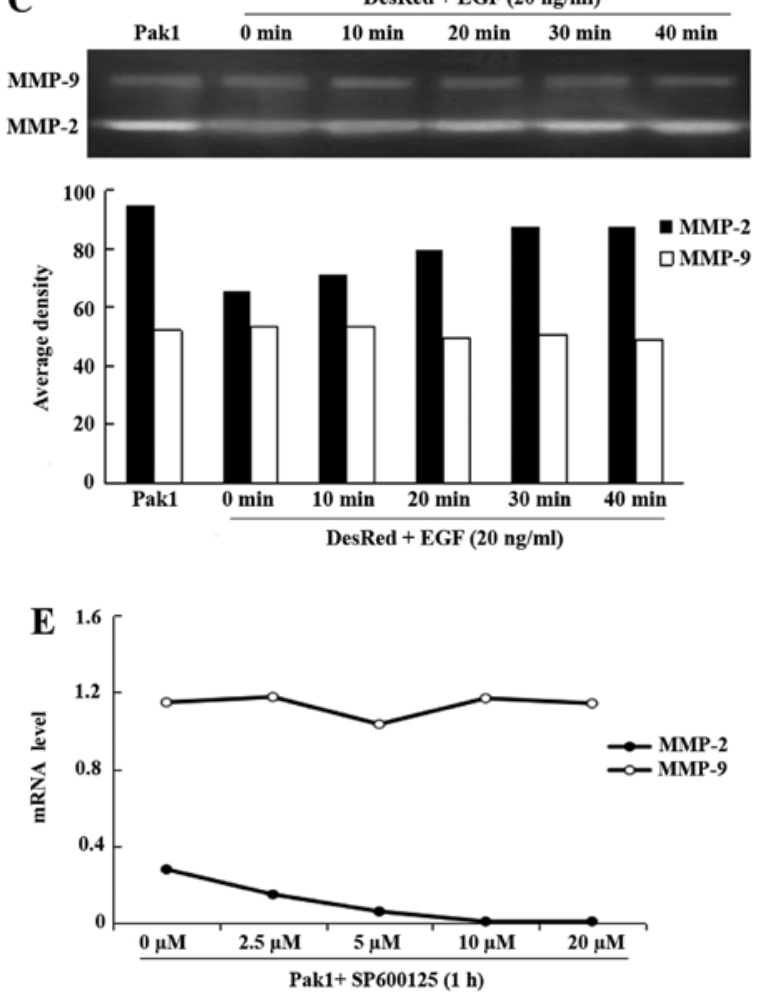
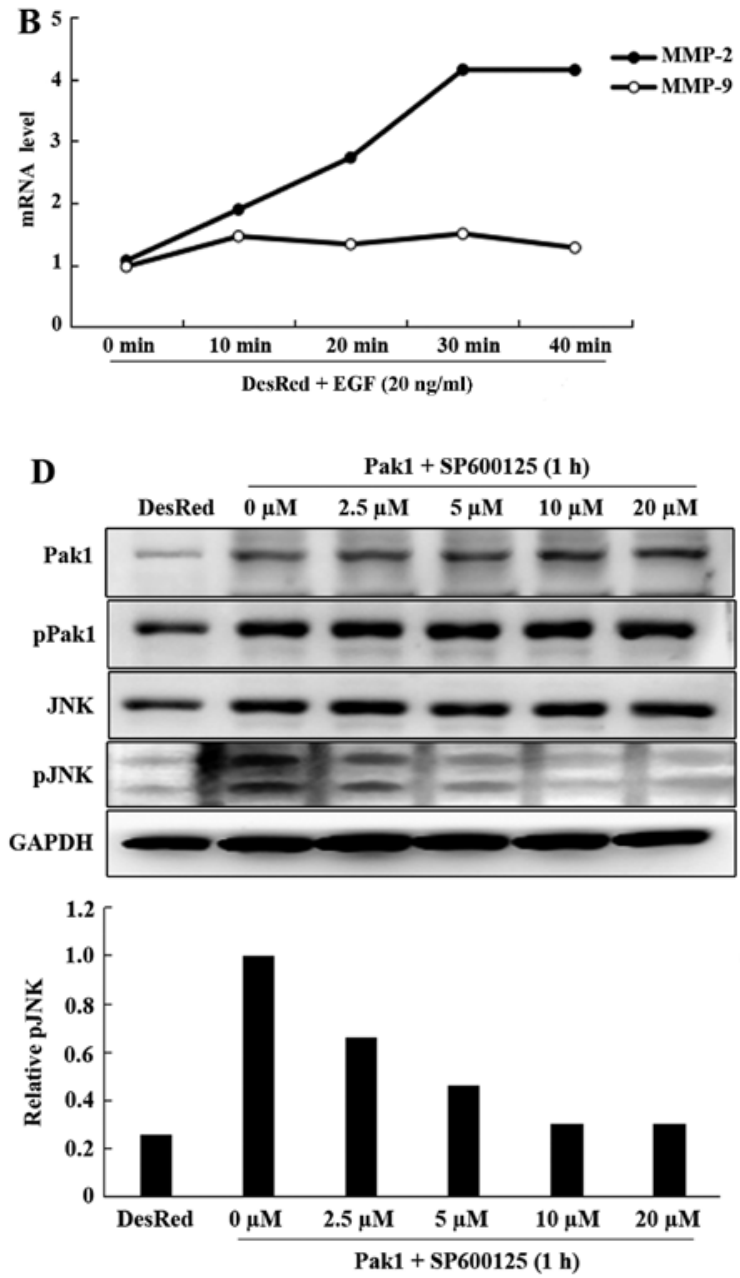

F
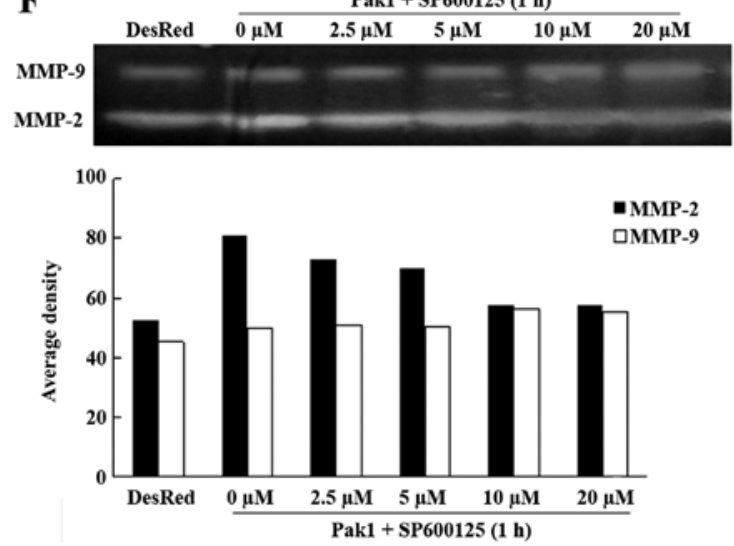

Figure 3. JNK mediates Pak1-induced upregulation of MMP-2 in human MKN45 gastric cancer cells. (A) MKN45-DsRed2 cells were serum starved and treated with EGF $(20 \mathrm{ng} / \mathrm{ml})$ for various times as indicated and were subsequently lysed. Western blot analysis of Pak1, phospho-Pak1, JNK and phospho-JNK (top panel). Quantification of phospho-JNK at various times (bottom panel). (B) MKN45-DsRed2 cells were serum starved and treated with EGF (20 ng/ml) for various times as indicated, MMP-2 and MMP-9 mRNA were subsequently evaluated using quantitative real-time RT-PCR. (C) MKN45-DsRed2 cells were serum starved and treated with EGF $(20 \mathrm{ng} / \mathrm{ml})$ for various times as indicated. Gelatin zymography assay results of MMP-2 and MMP-9 activities (top panel), Quantification of MMP-2 and MMP-9 activities (bottom panel). (D) MKN45-Pak1 cells were treated with increasing concentrations of SP600125 for $1 \mathrm{~h}$ and were subsequently lysed. Western blot analysis of Pak1, phospho-Pak1, JNK and phospho-JNK (top panel). Quantification of phospho-JNK at various concentrations (bottom panel). (E) MKN45-Pak1 cells were treated with increasing concentrations of SP600125 for $1 \mathrm{~h}$, MMP-2 and MMP-9 mRNA were subsequently evaluated using quantitative real-time RT-PCR. (F) MKN45-Pak1 cells were treated with increasing concentrations of SP600125 for $1 \mathrm{~h}$. Gelatin zymography assay results of MMP-2 and MMP-9 activities (top panel). Quantification of MMP-2 and MMP-9 activities (bottom panel). Pak1, p21-activated kinase 1; GC, gastric cancer; EGF, epidermal growth factor; JNK, c-Jun N-terminal kinase; MMP, matrix metalloproteinase.

Another invasion assay was performed with MKN45-Pak1 cells pretreated with the JNK inhibitor SP600125 at $10 \mu \mathrm{M}$ for $1 \mathrm{~h}$, and the MKN45-Pak1 cells were used as a control.
We observed that the MKN45-Pak1 cells pretreated with SP600125 invaded much more slowly than the control ( $73.50 \pm 5.68$ vs. $226.25 \pm 10.15, \mathrm{P}<0.01$; Fig. 4B). A decrease of 

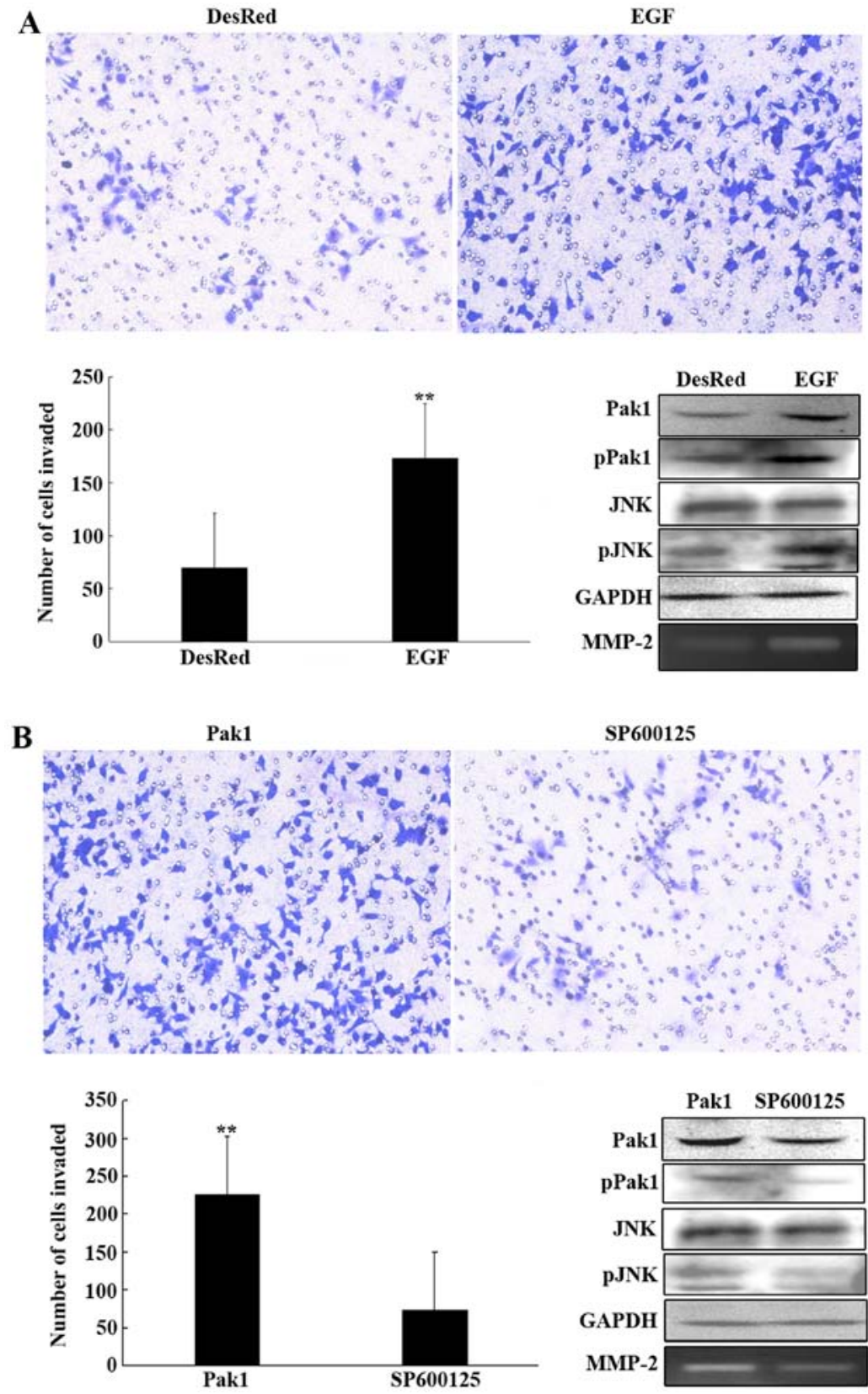

Figure 4. Upregulation of MMP-2 by Pak1 via the JNK pathway is important for human MKN45 gastric cancer cell invasion. (A) Representative images revealed the invasion assay for MKN45-DesRed2 cells incubated with EGF at $20 \mathrm{ng} / \mathrm{ml}$ for $30 \mathrm{~min}$, and MKN45-DesRed2 cells without EGF pretreatment used as a control (top panel). Invaded cells of 6 randomly selected fields were counted; ** $\mathrm{P}<0.01$ (left bottom panel). Western blot analysis of phospho-JNK and gelatin zymography assay of MMP-2 activities (right bottom panel). (B) Representative images revealed the invasion assay for MKN45-Pak1 cells pretreated with JNK inhibitor SP600125 at $10 \mu \mathrm{M}$ for $1 \mathrm{~h}$, and MKN45-Pak1 cells without SP600125 pretreatment used as a control (top panel). Invaded cells of 6 randomly selected fields were counted; ${ }^{* *} \mathrm{P}<0.01$ (left bottom panel). Western blot analysis of phospho-JNK and gelatin zymography assay of MMP-2 activities (right bottom panel). Pak1, p21-activated kinase 1; GC, gastric cancer; EGF, epidermal growth factor.

JNK phosphorylation and MMP-2 activity were determined using western blotting and gelatin zymography assay, respectively (Fig. 4B).

Expression of MMP-2 correlates with clinicopathological factors of human GC. To further confirm the biological functions of MMP-2 in GC cell invasion, MMP-2 mRNA was analyzed in 57 patient samples of GC by RT-PCR. The results revealed that MMP-2 mRNA expression in tumor tissue was significantly associated with the depth of invasion $(\mathrm{P}<0.01)$, lymph node status $(\mathrm{P}<0.01)$, distant metastasis $(\mathrm{P}<0.01)$ and tumor stage $(\mathrm{P}<0.01)$. However, the mRNA level of MMP-2 was independent of Lauren classification, tumor location, sex and age (Table I).

\section{Discussion}

Although, our previous study reported that Pak1 was overexpressed in GC, and obviously induced the invasive potential of GC cells (19), the exact molecular mechanism remained unclear. Upregulation of MMP-2 and MMP-9 is reported to degrade collagen IV, thus, leading to GC cell invasion and metastasis $(14,15)$. Thus, we wondered whether Pak1-stimulated GC cell invasion resulted from increased 
Table I. Association of MMP-2 expression with clinicopathological features of the GC cases.

\begin{tabular}{|c|c|c|c|}
\hline $\begin{array}{l}\text { Clinicopathological } \\
\text { features }\end{array}$ & $\mathrm{n}$ & $\begin{array}{c}\text { MMP-2 } \\
(\text { mean } \pm \text { SD })\end{array}$ & P-value \\
\hline Age (years) & & & 0.881 \\
\hline$>60$ & 27 & $74.11 \pm 11.26$ & \\
\hline$\leq 60$ & 30 & $76.42 \pm 10.35$ & \\
\hline Sex & & & 0.393 \\
\hline Male & 37 & $80.09 \pm 9.56$ & \\
\hline Female & 20 & $66.39 \pm 12.43$ & \\
\hline Tumor location & & & 0.699 \\
\hline Lower & 22 & $80.80 \pm 10.91$ & \\
\hline Middle & 26 & $67.49 \pm 12.25$ & \\
\hline Upper & 9 & $84.33 \pm 19.56$ & \\
\hline Lauren classification & & & 0.146 \\
\hline Intestinal & 35 & $66.53 \pm 9.50$ & \\
\hline Diffuse & 22 & $89.21 \pm 12.19$ & \\
\hline Depth of invasion & & & 0.000 \\
\hline $\mathrm{T} 1$ & 4 & $4.61 \pm 1.94$ & \\
\hline $\mathrm{T} 2$ & 18 & $43.43 \pm 12.96$ & \\
\hline $\mathrm{T} 3$ & 13 & $95.60 \pm 13.16$ & \\
\hline $\mathrm{T} 4$ & 22 & $102.20 \pm 9.87$ & \\
\hline Lymph node status & & & 0.000 \\
\hline N0 & 18 & $35.25 \pm 10.59$ & \\
\hline N1 & 21 & $74.65 \pm 12.10$ & \\
\hline $\mathrm{N} 2$ & 14 & $111.75 \pm 10.32$ & \\
\hline N3 & 4 & $131.19 \pm 25.49$ & \\
\hline Distant metastasis & & & 0.006 \\
\hline M0 & 52 & $68.95 \pm 7.74$ & \\
\hline M1 & 5 & $141.20 \pm 4.98$ & \\
\hline Stage & & & 0.000 \\
\hline $\mathrm{I}$ & 13 & $10.29 \pm 2.23$ & \\
\hline II & 7 & $28.93 \pm 16.19$ & \\
\hline III & 23 & $102.71 \pm 7.33$ & \\
\hline IV & 13 & $120.30 \pm 12.57$ & \\
\hline
\end{tabular}

MMP, matrix metalloproteinase; GC, gastric cancer.

levels of MMP-2 and MMP-9. MMP-2 and MMP-9 expression are reported to be regulated at many levels, including gene activation, mRNA stability, proenzyme activation, and inactivation by endogenous inhibitors in a complex fashion by numerous oncogene and tumor suppressor pathways and conditions of hypoxia (20-23). In the present study, we demonstrated that ectopic expression of Pak1 in MKN45 GC cells significantly increased MMP-2 mRNA expression, whereas specific knockdown of endogenous Pak1 in MKN45 cells sharply decreased the mRNA level of MMP-2, which was not due to changes in mRNA stabilities. We further revealed that upregulation of Pak1 expression directly induced MMP-2 activity, whereas downregulation of Pak1 expression decreased MMP-2 activity. However, Pak1 had no influence on MMP-9 mRNA expression and activity in MKN45 cells.

Activation of MMPs is regulated by physiological inhibitors, TIMPs (24). TIMPs not only directly inhibit MMPs, but also form complexes with MMPs to control activation and stability of MMPs $(18,25)$. Four different TIMP species have been identified as TIMP-1, TIMP-2, TIMP-3 and TIMP-4. TIMP binds to MMP in a 1:1 stoichiometric ratio. Injection of AdTIMP-2 into preestablished tumors resulted in the significant decreased metastasis of LLC tumors by $>90 \%$ (26), which emphasizes the importance of endogenous regulation of MMP activity by TIMPs. In the present study, we further detected the protein levels of TIMPs in the MKN45 cells with Pak1 overexpression and MKN45 cells with special knockdown of Pak1, which surprisingly demonstrated that Pak1 had no influence on the regulation of TIMPs. The results revealed that activation of MMP-2 was not related to a decrease in the specific inhibitor of TIMP-1, TIMP-2, TIMP-3 and TIMP-4 in MKN45 cells. There was, however, an increase in MMP/TIMP ratios, which is also in favor of ECM degradation.

Accumulating evidence has been dedicated to exploring the molecular mechanisms involved in the upregulation of cancer development. Mitogen-activated protein kinases (MAPKs) include 3 major subfamilies: the extracellular signal-regulated kinases (ERKs), the p38 MAPKs and the c-Jun N-terminal kinases (JNKs) (27). JNK signaling was revealed to regulate MMP-2 and MMP-9 production, which promoted invasiveness in human gliomas, colon, ovarian and prostate cancer (28-31). Our previous data revealed that activation of JNK was involved in Pak1 regulation of GC cell invasiveness (19). Thus, we hypothesized that Pak1 may induce the invasion of GC cells through the JNK-MMP-2 signaling pathway. Consistent with this hypothesis, we offered the evidence for the first time, that Pak1 increased MMP-2 mRNA expression and activity through a JNK-dependent pathway in MKN45 GC cells. In addition, higher invasive properties were also revealed in MKN45 cells with activation of JNK-MMP-2 by Pak1 overexpression. In the present study, we also confirmed that MMP-2 mRNA expression was significantly associated with aggressive progression of GC (depth of invasion, lymph node status, distant metastasis and tumor stage), mirroring previous studies (13-17).

One of the novel findings of the present study, is the demonstration that Pak1 is a direct transcriptional activator of MMP-2 synthesis. In the present study, we demonstrated that Pak1 increased MMP-2 mRNA expression and activity. This is the first study to reveal that MMP-2 is a target gene of Pak1 activation. Moreover, our results identified a JNK signaling pathway that mediates Pak1-stimulated MMP-2 expression, activity and cell invasion.

In summary, we demonstrated that Pak1 activates JNK1/2 kinase, and then increases MMP-2 mRNA expression and activity, which further promotes cellular invasion in human GC cells.

\section{Acknowledgements}

The present study was supported by grants from the Xiamen Science and Technology Projects (3502Z20124014). 


\section{References}

1. Tsugane S and Sasazuki S: Diet and the risk of gastric cancer: Review of epidemiological evidence. Gastric Cancer 10: 75-83, 2007.

2. Kumar R, Gururaj AE and Barnes CJ: p21-activated kinases in cancer. Nat Rev Cancer 6: 459-471, 2006.

3. Kumar R and Vadlamudi RK: Emerging functions of p21-activated kinases in human cancer cells. J Cell Physiol 193: $133-144,2002$.

4. Schürmann A, Mooney AF, Sanders LC, Sells MA, Wang HG, Reed JC and Bokoch GM: p21-activated kinase 1 phosphorylates the death agonist bad and protects cells from apoptosis. Mol Cell Biol 20: 453-461, 2000.

5. Vadlamudi RK, Adam L, Wang RA, Mandal M, Nguyen D, Sahin A, Chernoff J, Hung MC and Kumar R: Regulatable expression of p21-activated kinase-1 promotes anchorage-independent growth and abnormal organization of mitotic spindles in human epithelial breast cancer cells. J Biol Chem 275 36238-36244, 2000

6. Narumiya $\mathrm{S}$, Ishizaki $\mathrm{T}$ and Watanabe $\mathrm{N}$ : Rho effectors and reorganization of actin cytoskeleton. FEBS Lett 410: 68-72, 1997.

7. O'Sullivan GC, Tangney M, Casey G, Ambrose M, Houston A and Barry OP: Modulation of p21-activated kinase 1 alters the behavior of renal cell carcinoma. Int J Cancer 121: 1930-1940, 2007.

8. Ching YP, Leong VY, Lee MF, Xu HT, Jin DY and Ng IO: $\mathrm{P} 21$-activated protein kinase is overexpressed in hepatocellular carcinoma and enhances cancer metastasis involving c-Jun NH2-terminal kinase activation and paxillin phosphorylation. Cancer Res 67: 3601-3608, 2007.

9. Yoon WH, Jung YJ, Kim TD, Li G, Park BJ, Kim JY, Lee YC Kim JM, Park JI, Park HD, et al: Gabexate mesilate inhibits colon cancer growth, invasion, and metastasis byreducing matrix metalloproteinases and angiogenesis. Clin Cancer Res 10 4517-4526, 2004.

10. Coussens LM, Fingleton B and Matrisian LM: Matrix metalloproteinase inhibitors and cancer: Trials and tribulations. Science 295: 2387-2392, 2002.

11. Ellerbroek SM and Stack MS: Membrane associated matrix metalloproteinases in metastasis. Bioessays 21: 940-949, 1999.

12. Liotta LA and Stetler-Stevenson WG: Tumor invasion and metastasis: An imbalance of positive and negative regulation. Cancer Res 51 (Suppl 18): 5054s-5059s, 1991.

13. Nomura H, Fujimoto N, Seiki M, Mai M and Okada Y: Enhanced production of matrix metalloproteinases and activation of matrix metalloproteinase 2 (gelatinase A) in human gastric carcinomas. Int J Cancer 69: 9-16, 1996.

14. Torii A, Kodera Y, Uesaka K, Hirai T, Yasui K, Morimoto T, Yamamura Y, Kato T, Hayakawa T, Fujimoto N, et al: Plasma concentration of matrix metalloproteinase 9 in gastric cancer. $\mathrm{Br}$ J Surg 84: 133-136, 1997.

15. Kubben FJ, Sier CF, van Duijn W, Griffioen G, Hanemaaijer R, van de Velde CJ, van Krieken JH, Lamers CB and Verspaget HW: Matrix metalloproteinase-2 is a consistent prognostic factor in gastric cancer. Br J Cancer 94: 1035-1040, 2006.

16. Sier CFM, Kubben FJGM, Ganesh S, Heerding MM, Griffioen G, Hanemaaijer R, van Krieken JH, Lamers CB and Verspaget HW: Tissue levels of matrix metalloproteinases MMP-2 and MMP-9 are related to the overall survival of patients with gastric carcinoma. Br J Cancer 74: 413-417, 1996.
17. Shen $\mathrm{W}, \mathrm{Xi} \mathrm{H}$, Wei B and Chen L: The prognostic role of matrix metalloproteinase 2 in gastric cancer: A systematic review with meta-analysis. J Cancer Res Clin Oncol 140: 1003-1009, 2014.

18. Gomez DE, Alonso DF, Yoshiji H and Thorgeirsson UP: Tissue inhibitors of metalloproteinases: Structure, regulation and biological functions. Eur J Cell Biol 74: 111-122, 1997.

19. Li LH, Luo Q, Zheng MH, Pan C, Wu GY, Lu YZ, Feng B, Chen XH and Liu BY: P21-activated protein kinase 1 is overexpressed in gastric cancer and induces cancer metastasis. Oncol Rep 27: 1435-1442, 2012

20. Sehgal I and Thompson TC: Novel regulation of type IV collagenase (matrix metalloproteinase-9 and -2) activities by transforming growth factor- $\beta 1$ in human prostate cancer cell lines. Mol Biol Cell 10: 407-416, 1999.

21. Baruch RR, Melinscak H, Lo J, Liu Y, Yeung O and Hurta RA: Altered matrix metalloproteinase expression associated with oncogene-mediated cellular transformation and metastasis formation. Cell Biol Int 25: 411-420, 2001.

22. Nair RR and Boyd DD: Expression cloning of novel regulators of $92 \mathrm{kDa}$ type IV collagenase expression. Biochem Soc Trans 33: 1135-1136, 2005

23. Muñoz-Nájar UM, Neurath KM, Vumbaca F and Claffey KP; MunozNajar UM: Hypoxia stimulates breast carcinoma cell invasion through MT1-MMP and MMP-2 activation. Oncogene 25: 2379-2392, 2006.

24. Li YY, McTiernan CF and Feldman AM: Interplay of matrix metalloproteinases, tissue inhibitors of metalloproteinases and their regulators in cardiac matrix remodeling. Cardiovasc Res 46: 214-224, 2000.

25. Goldberg GI, Marmer BL, Grant GA, Eisen AZ, Wilhelm S and He CS: Human 72-kilodalton type IV collagenase forms a complex with a tissue inhibitor of metalloproteases designated TIMP-2. Proc Natl Acad Sci USA 86: 8207-8211, 1989.

26. Li H, Lindenmeyer F, Grenet C, Opolon P, Menashi S, Soria C, Yeh P, Perricaudet M and Lu H: AdTIMP-2 inhibits tumor growth, angiogenesis, and metastasis, and prolongs survival in mice. Hum Gene Ther 12: 515-526, 2001

27. Marino M, Galluzzo P and Ascenzi P: Estrogen signaling multiple pathways to impact gene transcription. Curr Genomics 7: 497-508, 2006.

28. Hu B, Jarzynka MJ, Guo P, Imanishi Y, Schlaepfer DD and Cheng SY: Angiopoietin 2 induces glioma cell invasion by stimulating matrix metalloprotease 2 expression through the alphavbetal integrin and focal adhesion kinase signaling pathway. Cancer Res 66: 775-783, 2006.

29. Hsu HH, Hu WS, Lin YM, Kuo WW, Chen LM, Chen WK, Hwang JM, Tsai FJ, Liu CJ and Huang CY: JNK suppression is essential for 17 $\beta$-Estradiol inhibits prostaglandin E2-Induced uPA and MMP-9 expressions and cell migration in human LoVo colon cancer cells. J Biomed Sci 18: 61-72, 2011.

30. Cheung LW, Leung PC and Wong AS: Gonadotropin-releasing hormone promotes ovarian cancer cell invasiveness through c-Jun $\mathrm{NH}_{2}$-terminal kinase-mediated activation of matrix metalloproteinase (MMP)-2 and MMP-9. Cancer Res 66: 10902-10910, 2006.

31. Chien CS, Shen KH, Huang JS, Ko SC and Shih YW: Antimetastatic potential of fisetin involves inactivation of the PI3K/Akt and JNK signaling pathways with downregulation of MMP-2/9 expressions in prostate cancer PC-3 cells. Mol Cell Biochem 333: 169-180, 2010. 